\title{
Periodic traveling compression regions during quiet geomagnetic conditions and their association with ground $\mathrm{Pi} 2$
}

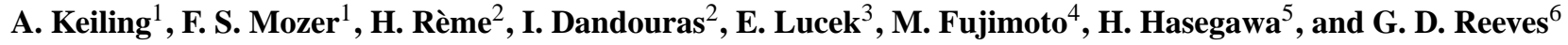 \\ ${ }^{1}$ Space Sciences Laboratory, University of California at Berkeley, CA 94720, USA \\ ${ }^{2}$ Centre d'Etude Spatiale des Rayonnements, Toulouse, 31028 cedex 4, France \\ ${ }^{3}$ Space and Atmospheric Physics, Imperial College, London, SW7 2BZ, UK \\ ${ }^{4}$ Earth and Planetary Sciences, Tokyo Institute of Technology, Tokyo, Japan \\ ${ }^{5}$ Institute of Space and Astronautical Science, JAXA, Sagamihara, Japan \\ ${ }^{6}$ Los Alamos National Laboratory, Los Alamos, NM 87545, USA
}

Received: 25 July 2007 - Revised: 29 July 2008 - Accepted: 3 September 2008 - Published: 21 October 2008

\begin{abstract}
Recently, Keiling et al. (2006) showed that periodic ( $\sim 90 \mathrm{~s}$ ) traveling compression regions (TCRs) during a substorm had properties of $\mathrm{Pi} 2$ pulsations, prompting them to call this type of periodic TCRs "lobe Pi2". It was further shown that time-delayed ground Pi2 had the same period as the lobe Pi2 located at $16 R_{E}$, and it was concluded that both were remotely driven by periodic, pulsed reconnection in the magnetotail. In the study reported here, we give further evidence for this association by reporting additional periodic TCR events (lobe Pi2s) at $18 R_{E}$ all of which occurred in succession during a geomagnetically very quiet, non-substorm period. Each quiet-time periodic TCR event occurred during an interval of small $H$-bay-like ground disturbance $(<40 \mathrm{nT})$. Such disturbances have previously been identified as poleward boundary intensifications (PBIs). The small $H$ bays were superposed by Pi2s. These ground Pi2s are compared to the TCRs in the tail lobe (Cluster) and both magnetic pulsations and flow variations at $9 R_{E}$ inside the plasma sheet (Geotail). The main results of this study are: (1) Further evidence is given that periodic TCRs in the tail lobe at distances of $18 R_{E}$ and ground $\mathrm{Pi} 2$ are related phenomena. In particular, it is shown that both had the same periodicity and occurred simultaneously (allowing for propagation time delays) strongly suggesting that both had the same periodic source. Since the TCRs were propagating Earthward, this source was located in the outer magnetosphere beyond $18 R_{E}$. (2) The connection of periodic TCRs and ground $\mathrm{Pi} 2$ also exists during very quiet geomagnetic conditions with PBIs present in addition to the previous result (Keiling et al., 2006) which showed this connection during substorms. (3) Combining (1) and (2), we conclude that the frequency of
\end{abstract}

Correspondence to: A. Keiling

(keiling@ssl.berkeley.edu)
PBI-associated Pi2 is controlled in the outer magnetosphere as opposed to the inner magnetosphere. We propose that this mechanism is pulsed reconnection based on previous results which combined modeled results and observations of substorm-related periodic TCRs and ground Pi2. (4) We show that TCRs with small compression ratios $(\Delta \mathrm{B} / \mathrm{B}<1 \%)$ can be useful in the study of magnetotail dynamics and we argue that other compressional fluctuations with $\Delta \mathrm{B} / \mathrm{B}<1 \%$ (without having all of the characteristic signatures of TCRs) seen in the tail lobe could possibly be related to the same mechanism that generates TCR with $\Delta \mathrm{B} / \mathrm{B}>1 \%$ (which are more commonly studied). (5) Finally, it is noted that both quiet time and substorm-related periodic TCRs had remarkably similar periods in spite of the drastically different geomagnetic conditions prevailing during the events which poses the important question of what causes this periodicity under these different conditions.

Keywords. Magnetospheric physics (Magnetospheric configuration and dynamics; Magnetotail; Storms and substorms)

\section{Introduction}

For a long time space observations of the Pi2 phenomenon geomagnetic oscillations with periods between 40 and $150 \mathrm{~s}$ and durations of only a few wave periods - had been confined to the inner magnetosphere $\left(<10 R_{E}\right)$ and had excluded the lobe regions which suggested that the mechanism controlling the Pi2 frequency was confined to the inner magnetosphere. The first indication that processes beyond $10 R_{E}$ could also directly control the frequency of ground $\mathrm{Pi} 2$ was given in the study by Kepko and Kivelson (1999), who correlated

Published by Copernicus Publications on behalf of the European Geosciences Union. 
velocity variations of plasma flows with magnetic field waveforms of ground Pi2. Technically, the flow bursts are not Pi2 pulsations because Pi2s are defined as oscillations of the geomagnetic field. The first geomagnetic pulsations in the tail lobe which can be termed Pi2 pulsations were recently reported by Kim et al. (2005a) and Keiling et al. (2006). The lobe Pi2s recorded by the Polar satellite at $<9 R_{E}$ (Kim et al., 2005a) were correlated with ground Pi2s. Based on the wave properties of the lobe Pi2s, Kim et al. argued that the plasmaspheric virtual resonance mechanism (Lee and Lysak, 1999) generated these Pi2s, which implies that the mechanism for the Pi2 frequency still lies within the inner magnetosphere. In contrast, Keiling et al. (2006) reported a substorm-related Pi2 event in the tail lobe at an unusually large distance of $16 R_{E}$ using the Cluster spacecraft. This lobe $\mathrm{Pi} 2$ propagated toward Earth at a speed of $\sim 700 \mathrm{~km} / \mathrm{s}$. Location and propagation direction ruled out that the $\mathrm{Pi} 2$ was generated in the inner magnetosphere. Instead, it was argued that the time variation of reconnection (i.e. pulsed reconnection) beyond $16 R_{E}$ was actively controlling the Pi2 frequency of both the lobe Pi2 and the time-delayed identical ground Pi2. The association with pulsed reconnection was based on the observation that the lobe Pi2 comprised a series of traveling compression regions (TCRs) or nightside flux transfer events (NFTE), which are thought to be the remote signature of reconnection (e.g. Slavin et al., 1984, 2003a; Sergeev et al., 1993; Taguchi et al., 1998). It was thus further suggested by Keiling et al. that one type of substorm-related ground $\mathrm{Pi} 2$ is associated with TCRs/NFTEs.

Two scenarios currently exist for the generation of TCRs, namely via flux ropes inside the plasma sheet requiring multiple $\mathrm{X}$-lines, and via impulsive reconnection from possibly a single X-line. The latter scenario has also been called NFTE (Sergeev et al., 1992). It is noted that the term TCR comprises both scenarios. Sergeev et al. (2005) suggested that in addition to the topological difference between both scenarios, there is a difference in the bipolar variations of $B_{z}$ (GSM) of both magnetic structures. Whereas in the multiple$\mathrm{X}$-line scenario the bipolar variations are symmetric, they are asymmetric (small deflection followed by a larger opposite deflection) in the impulsive reconnection scenario. Both scenarios show the characteristic compressional increase in the magnetic field strength. For the remaining part of this manuscript we will omit the term NFTE and use only the more general term TCR. Initially, TCRs were recorded in the distant tail while traveling away from Earth together with plasmoids (Slavin et al., 1984). Earthward traveling TCRs in the near-tail were recently reported (e.g. Slavin et al., 2003b; Sergeev et al., 2005). TCRs often occur as individual events but can sometimes occur as a series of multiple TCRs with temporal separations of less than $150 \mathrm{~s}$ (Slavin et al., 2005). Examinations of TCRs and substorm phases show that TCRs occur during all phases of substorm (Slavin et al., 1993, 2005). Moldwin and Hughes (1994) reported TCRs during very quiet geomagnetic conditions during small "high-latitude substorms" which might now be more appropriately called poleward boundary intensifications (PBIs) see further discussion below.

A recent development in $\mathrm{Pi} 2$ research has been the discovery of Pi2 during very quiet geomagnetic conditions (Sutcliffe, 1998; Sutcliffe and Lyons, 2002). Sutcliffe and Lyons (2002) showed for one event their association with PBIs on the basis of ground magnetometer data. Although PBIs are largely defined by their optical signature (e.g. Lyons et al., 1999), they also show small bay-like perturbations in the $H$ component of ground magnetometer data. Kim et al. (2005b) investigated the same event and proposed that the source of the Pi2 which establishes the frequency and duration was in the magnetotail albeit no direct observational evidence was given. The study presented here extends these results by showing direct evidence in the tail that PBI-associated ground $\mathrm{Pi} 2$ during very quiet times are indeed driven by a source in the tail, and we propose reconnection as the source on the basis of their association with TCRs which were simultaneously recorded in the tail lobes at $18 R_{E}$ by the Cluster spacecraft. This association assumes the conventional understanding that TCRs are the result of impulsive or multiple$\mathrm{X}$-line tail reconnection. Hence, we also extend the finding of Keiling et al. (2006) - who showed one-to-one correlations of periodic TCRs and the individual pulses of ground Pi2 at substorm onset - by showing the same correspondence of periodic TCRs and ground $\mathrm{Pi} 2$ during very quiet geomagnetic conditions.

\section{Observations}

\subsection{Cluster observations}

Two 2-h intervals of Cluster and Geotail data recorded on 24 August 2001 and 8 September 2002 are shown in Fig. 1. The first interval constitutes a period of very quiet geomagnetic activity albeit small bay-like disturbances were recorded in ground magnetometer data (shown in Sects. 2.2 and 2.5). The Cluster spacecraft were located at about $X_{\mathrm{GSE}}=-18 R_{E}$ in the Northern Hemisphere. Following the onset of the first small ground-bay disturbance (dashed line) are three short intervals in the Cluster data which show periodic variations in $B_{z}$ (Fig. 1b) and $B_{x}$ denoted as events A, B, and C. Figure 2 shows expanded views of the magnetic field of these events. Whereas event $\mathrm{C}$ is clearly discernable in the non-detrended data (panels a-d), events A and B are more difficult to identify because of other superposing small-amplitude contributions. Therefore, the same magnetic field components are shown in panels (e) to (g) after applying a band-pass filter $(40 \mathrm{~s}, 150 \mathrm{~s})$ which now clearly shows event B. Event A is still speculative but we also consider it here as an event in light of the ground magnetometer data shown in Sect. 2.5. All three events were recorded while the Cluster spacecraft were in the tail lobe as determined from the ion density and 

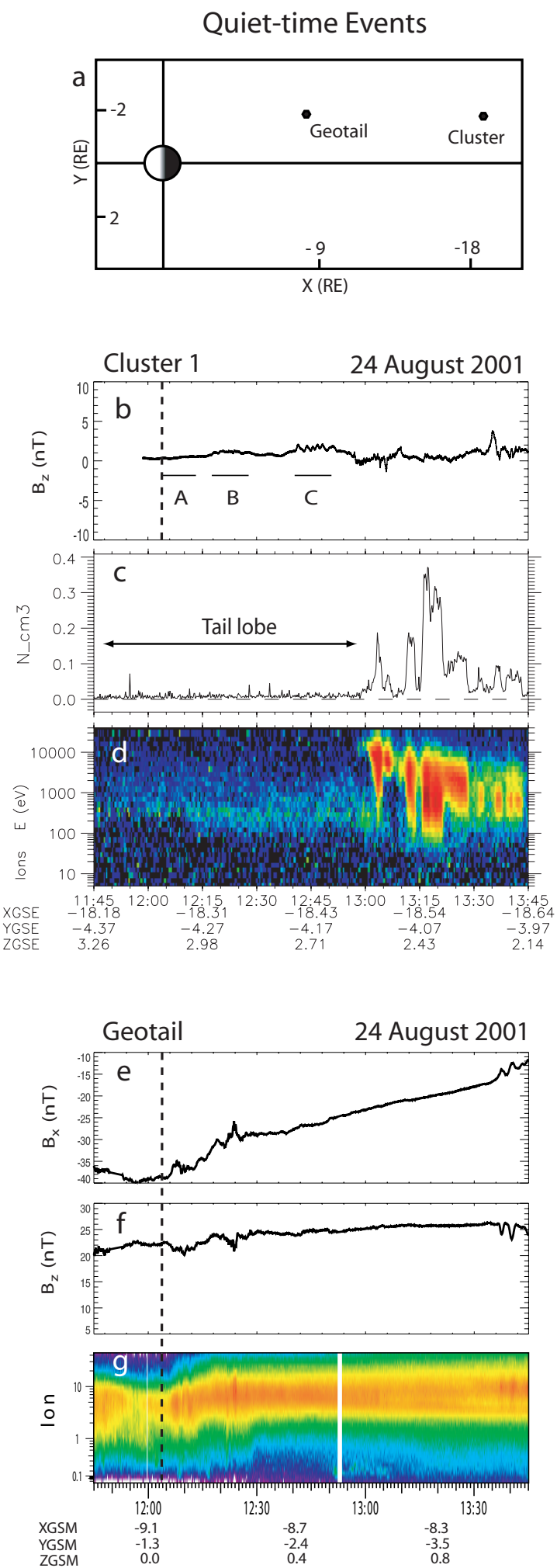

Substorm Event

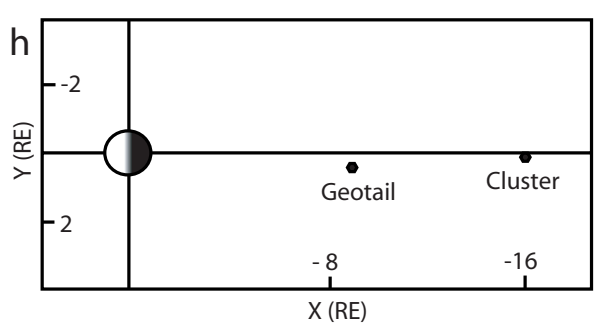

\section{Cluster $1 \quad 8$ September 2002}
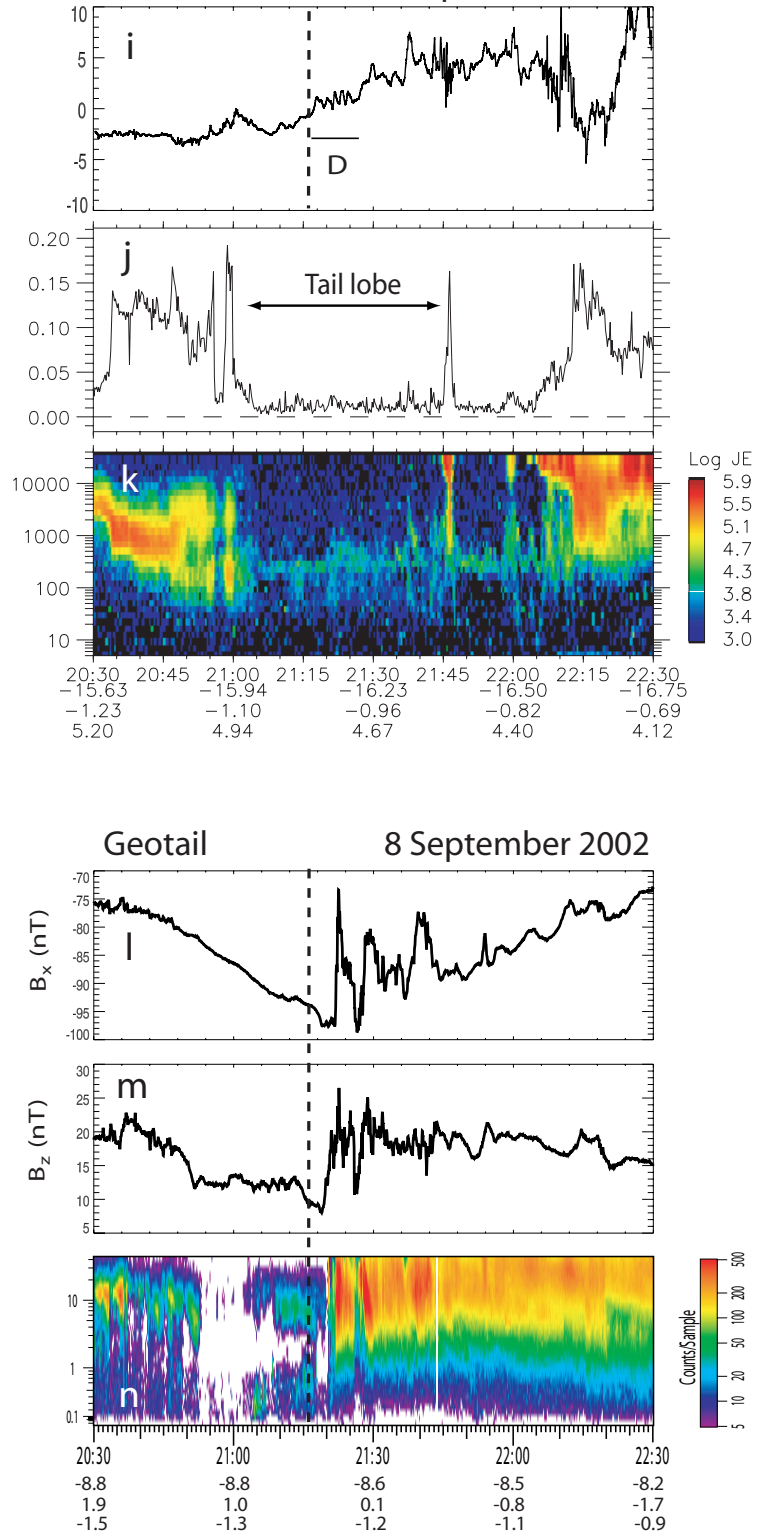

Fig. 1. Overview plots of four events (labeled A, B, C, and D) showing Cluster and Geotail data and their locations in the X-Y plane: (a-g) 24 August 2001 and (h-n) 8 September 2002. Cluster was inside the tail lobe during all events. Geotail was located in the plasma sheet during the 24 August events and near the outer edge of the plasma sheet during the 8 September event. 

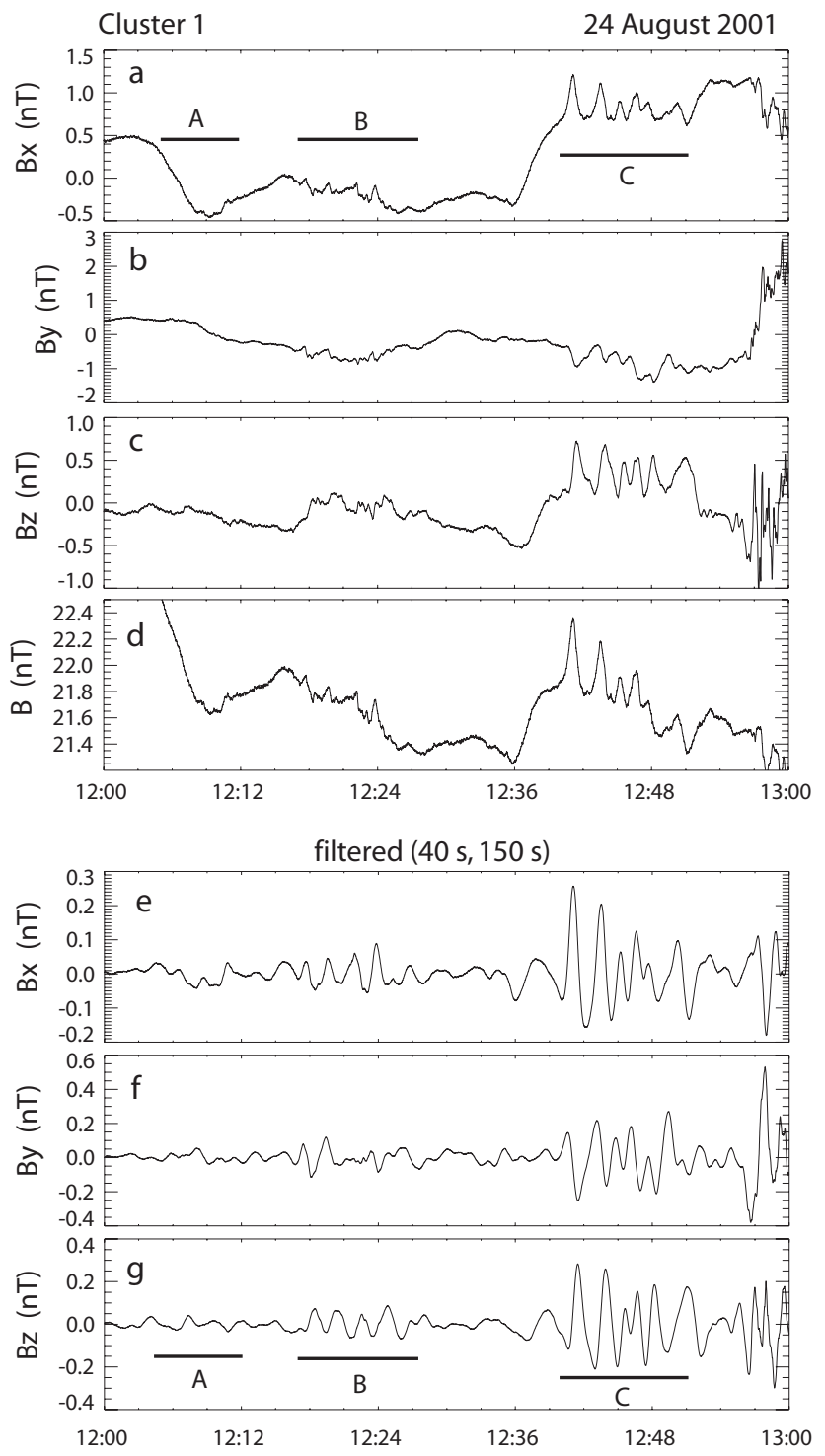

Fig. 2. Magnetic field data from Cluster 1 encompassing three events (A, B, and C) on 24 August 2001. (a-d) Data were averaged (4s); (e-f) Data were averaged (40 s) and detrended (150 s).

the ion energy-time spectrogram (HIA instrument) (Fig. 1c and d).

Figure 3 shows the three magnetic field components (filtered) and the total magnetic field for each event in the order (C, B, A) of being closest to the plasma sheet. Events B and $\mathrm{C}$ are identified as periodic TCRs; event $\mathrm{C}$ was previously reported by Slavin et al. (2005) who also identified this event as several TCRs. The compressional variations (dashed lines) and a phase shift between $B_{z}$ and $B_{x}$ are expected signatures of TCRs. It is noted, however, that the phase shifts are not the same for all pulses within each event. For example, the fourth pulse (from left) of event $\mathrm{C}$ shows almost no phase shift. In some cases, e.g. third pulse of event B and $\mathrm{C}$, the peak in $B_{x}$ occurs closer to the minimum of $B_{z}$ but still on the rising slope. This asymmetry can be due to varying shapes of the leading and trailing edges of flux ropes or reconnection pulses. According to Sergeev et al. (2005), this signature might be typical for NFTEs and can also be found in the event $\mathrm{D}$ (shown below). Moreover, the distance of the TCR observation point from the plasma sheet has effects on the relationship between $B_{x}$ and $B_{z}$ due to varying degrees of tail lobe flaring (Taguchi et al., 1997). For event A we only find a clear TCR-like signature in the magnetic compression, best seen in the filtered $B_{x}$ since the total $B$ is much larger than the compression, $\Delta B$. The phase shift relationship between $B_{x}$ and $B_{z}$ is not conclusive. However, one has to consider that this event is recorded farther away from the plasma sheet than the other two events and therefore has a very small compression ratio, $\Delta B / B$, of $\sim 0.09 \%$ in comparison to $0.24 \%$ and $1.37 \%$ for events $B$ and $C$, respectively. Typically, in the literature only TCRs with larger compression ratios are considered. For example, in the survey of Cluster data by Slavin et al. (2005) only TCRs with compression ratios greater than $\sim 1 \%$ were considered. Although event A does not show all characteristic signatures of TCR, it is suggestive that it is caused by the same mechanism as B and $\mathrm{C}$ because of its occurrence at the onset of a bay-like signature in the ground magnetometer data (see Sect. 2.5). Below we will show that such bays are indeed associated with TCRs in the tail lobe by using the events B and C. It is, however, beyond the scope of this study to prove that event $\mathrm{A}$ is also caused by a traveling plasma sheet bulge (which is the source of a TCR) which would require simultaneous observations in the plasma sheet at Cluster's distance of $\sim 18 R_{E}$ in the tail. Hence, we do not make this assertion but simply suggest it here (see Sect. 2.5 for further discussion of this speculation).

For each event all four spacecraft show very similar but slightly time-delayed pulses in $B_{x}$ which generally allows the determination of the speed of the propagating disturbance. Cross-correlation analysis on the magnetic field among the four Cluster spacecraft shows that the TCRs of events B and C propagated towards Earth at a speed of at least $2000 \mathrm{~km} / \mathrm{s}$. The shortness of the time delays yielded a large uncertainty in the speed measurements which only allowed us to determine a lower limit on the speed (cf. Slavin et al., 2005). It was not possible to obtain any consistent estimate for event A.

Turning now to the substorm-related event $\mathrm{D}$ on 8 September 2002, Fig. 1i-n shows an overview plot using the same data quantities as for the quiet-time events. Cluster 1 $\left(X_{\mathrm{GSE}}=-16 R_{E}\right)$ left the plasma sheet at about 21:05 UT and remained on tail lobe field lines until 21:45 UT when it briefly reentered the plasma sheet (Fig. $1 \mathrm{j}$ and $\mathrm{k}$ ). While in the tail lobe, Cluster recorded a periodic TCR event (labeled $\mathrm{D}$ in Fig. 1i) which traveled towards Earth with a speed of $\sim 700 \mathrm{~km} / \mathrm{s}$ (as reported by Sergeev et al., 2005; Keiling et al., 2006) which is significantly smaller than for the TCR 
events on 24 August 2001. It has been demonstrated that the magnetic field signature (Fig. 4) of event D is consistent with the signature of TCR (Sergeev et al., 2005; Semenov et al., 2005). These periodic TCRs occurred during a substorm, or more precisely, it was the first signature of a substorm onset which then $30 \mathrm{~s}$ later was recorded on the ground. The plasma sheet encounters at $\sim 21: 45$ UT and $\sim 22: 05$ UT (i.e. after the periodic TCR event) show elevated ion energies ( $>20 \mathrm{keV}$ ) compared to the plasma sheet encounter (20:3021:00 UT) before the event. These energy increases are consistent with the fact that a substorm onset occurred in between the plasma sheet encounters.

The next section further describes the global geomagnetic conditions during both the quiet-time and substorm events. Geotail data shown in Fig. 1 (bottom) are discussed in Sect. 2.3.

\subsection{Interplanetary and geomagnetic conditions}

The magnetosphere was in a quiet state on 24 August 2001. The $K_{p}$ index was less than or equal to 1 and the AU/AL indices (quick-look version from the World Data Center for Geomagnetism, Kyoto) were less than $50 \mathrm{nT}$ for many hours before and during the event (Fig. 5a). Inspection of highlatitude ground magnetometer data from the Geophysical Institute Magnetometer Array (GIMA) in the vicinity of Cluster's footprints (Fig. 6) show a small bay disturbance at $\sim 12: 03$ UT (first dashed line from left) in the $H$ component $(<40 \mathrm{nT})$ followed by additional small bays (second and third dashed lines; see Arctic Village station). Such small bays are not associated with substorms but with PBIs (e.g. Sutcliffe and Lyons, 2002). No optical data were available at the time of these events to confirm the auroral signature of PBIs. In addition, each bay onset was followed by Pi2 activity. These bays and Pi2s are correlated with the events identified in the Cluster data (labels A, B, and C). A more detailed comparison of ground and space pulsations is presented in Sect. 2.5. In contrast, on 8 September 2002 both $K_{p}$ and AU/AL showed increased activity surrounding the event D (Fig. 5f). AU/AL activity started at around 20:00 UT and was preceded by many hours of quiescence. At 21:18 UT the expansion phase of a substorm began as seen in a sharp negative turning of the AL index (see dashed line) and as seen in individual ground stations (not shown here, see Keiling et al., 2006).

IMF and solar wind conditions (recorded by ACE and time-shifted to the Earth) are also shown in Fig. 5 for both time periods. ACE was located $\sim 226 R_{E}$ upstream in the solar wind. On 24 August 2001, the solar wind speed was of average magnitude and its density was a little bit below average. The IMF $B_{z}$ pointed mostly north with small amplitude $(\sim 2 \mathrm{nT})$ which correspond to very quiet conditions. Figure $5 \mathrm{~g}-\mathrm{j}$ shows the corresponding data for 8 September 2002. For a direct comparison the same scales on all quantities were chosen for both time periods. The IMF $B_{z}$ was
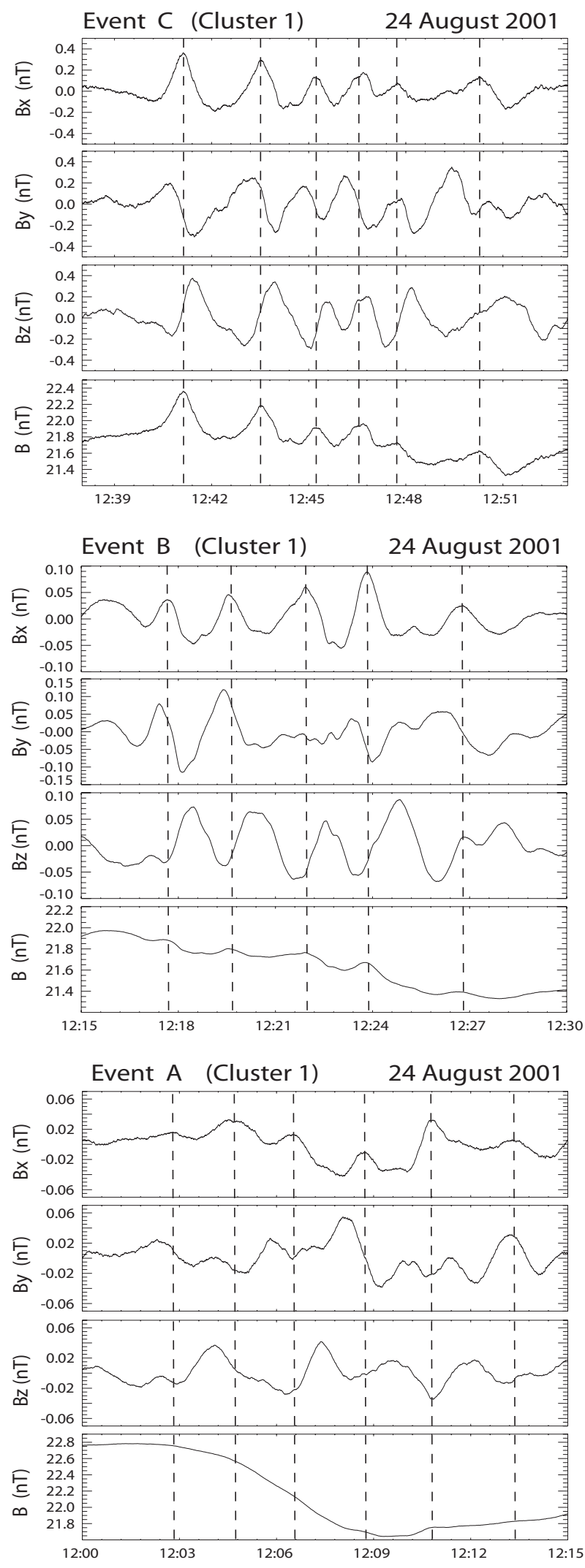

Fig. 3. Magnetic field data for events A, B, and C. The components $B_{x}, B_{y}$ and $B_{z}$ were averaged $(40 \mathrm{~s})$ and detrended ( $\left.150 \mathrm{~s}\right)$. Vertical dashed lines are visual aids. 


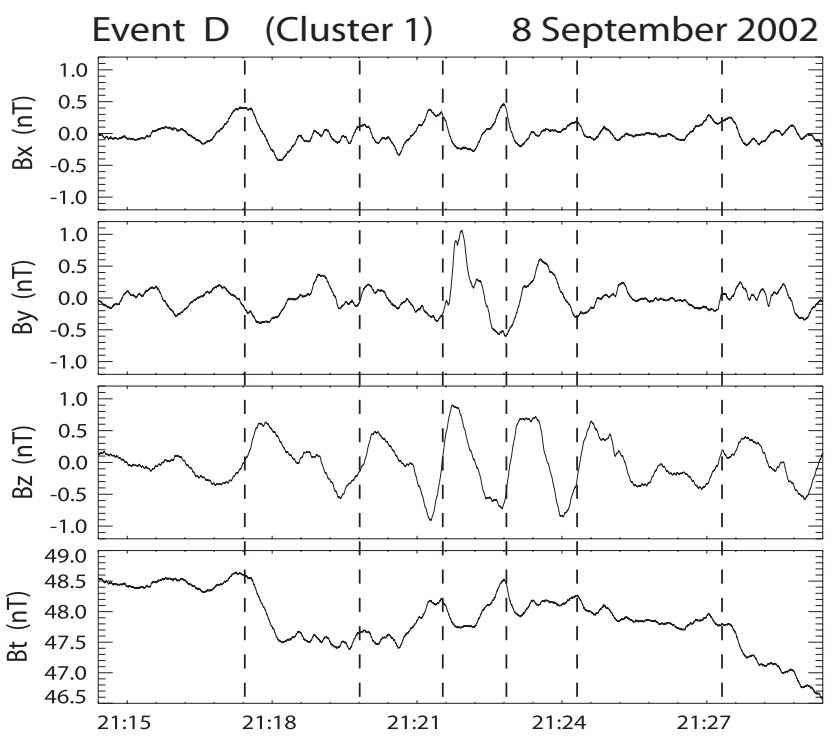

Fig. 4. Magnetic field data for event D. The components $B_{x}, B_{y}$ and $B_{z}$ were averaged $(40 \mathrm{~s})$ and detrended $(150 \mathrm{~s})$. Vertical dashed lines are visual aids.

north for many hours before the Cluster event (D) but larger $(<10 \mathrm{nT})$ than compared to the 24 August 2001 period. At 20:00 UT it turned south for a while and then north again. The southward turning almost coincided with the increase in AU/AL activity (growth phase) and the northward turning coincided with the substorm onset, which is a typical behavior for some substorms (Lyons, 1995). At the onset time (dashed line) solar wind density and speed were slightly higher than on 24 August 2001.

\subsection{Geotail observations}

Referring back to Fig. 1e-g, Geotail was inside the plasma sheet below the neutral sheet during the entire 2-h interval on 24 August 2001. At the time of the first small-bay ground onset (dashed line), the magnetic field showed enhanced fluctuations and the plasma energy (LEP instrument) increased. Both changes were most likely related to the small ground activation which, however, was not a substorm as shown above (Sect. 2.2). Data from the EPIC instrument also confirms an energization to higher energies $(>100 \mathrm{keV})$ at the onset time (not shown). During the interval Geotail was approaching the field reversal region as inferred from the slowly decreasing $\left|B_{x}\right|$. Hence, events A and B occurred while Geotail was closer to the outer edge of the plasma sheet than during event $\mathrm{C}$ when Geotail was located deeper inside the central plasma sheet, consistent with the reduced fine structure in both the magnetic field and the plasma data. However, it cannot be determined how close events A and B were to the outer edge of the plasma sheet since the lobe-PSBL interface was not crossed. Using $B_{z}$ of $\sim 24 \mathrm{nT}$ in the plasma sheet (Fig. 1f), Takada et al. (2006)'s estimates (their Fig. 3e) show that such a $B_{z}$ at a radial distance of $9 R_{E}$ corresponds to a thick current sheet. A thick current sheet is also expected considering the prevailing IMF condition (Sect. 2.2).

On 8 September 2002, Geotail was in the lobe at onset time of event $\mathrm{D}$ but was soon after engulfed by the expanding plasma sheet (Fig. 11-n). The magnetic field $\left(B_{z}\right)$ indicates a dipolarization at about 21:20 UT along with the plasma sheet expansion at which time the plasma sheet shows substorm-like energies $(>10 \mathrm{keV})$ for the bulk plasma. $\left|B_{x}\right|$ sharply drops on entering the plasma sheet and a few times thereafter, which is the expected diamagnetic response of increased plasma pressure in the plasma sheet. Prior to dipolarization, starting at $\sim 20: 15 \mathrm{UT},\left|B_{x}\right|$ shows a steady increase and $B_{z}$ a steady decrease both of which are indicative of a stretching process in the tail. These changes coincided with a period of negative IMF $B_{z}$ (Fig. $5 \mathrm{~g}$ ). All these signatures are consistent with the substorm growth phase. In Sergeev et al. (2005), the current sheet was further characterized by utilizing magnetic field data from Polar which happened to be located above the current sheet at approximately the same radial distance as Geotail. By calculating the difference between the $B_{x}$ components (model subtracted) above and below the current sheet, it was concluded that the current density underwent a strong growth up to substorm onset.

Additional Geotail data (velocity moments and filtered magnetic field) are shown in Sect. 2.5 in comparison with ground magnetometer data.

\subsection{Geosynchronous LANL satellites}

Figure 7 shows differential fluxes of energetic electrons from several geosynchronous LANL satellites. The MLT values of the satellites' locations are given in each panel. During the events (A and B), none of the LANL satellites were located near midnight where geosynchronous satellites are most likely to observe dispersionless particle injections. Nevertheless, the energetic particle data allow drawing conclusions on the activity level which are in agreement with the global conditions presented in Sect. 2.2. On 8 September 2002, all of the LANL satellites recorded an electron injection event with energy dispersion. This injection event was preceded by a decrease of electron fluxes which is consistent with the growth phase (Baker et al., 1981). Furthermore, 1990-085 and 1994-084 show signatures of drift effects (energy dispersion and MLT-dependent variations) in the eastward drifting electrons (e.g. Reeves et al., 1990; Sergeev et al., 1992a). We note that LANL-01A recorded some perturbation at $\sim 21: 05$ UT before the main event. It is likely that this perturbation is associated with the pseudo-breakup discussed by Sergeev et al. (2005).

On 24 August 2001 the electron fluxes at the two LANL satellites (1994-084 and LANL-97A) that recorded data (Fig. 7) were nearly unperturbed (flat line), showing that no substorm injection had occurred at or propagated to geosynchronous orbit. No injection was observed in the ion fluxes 
24 August 2001
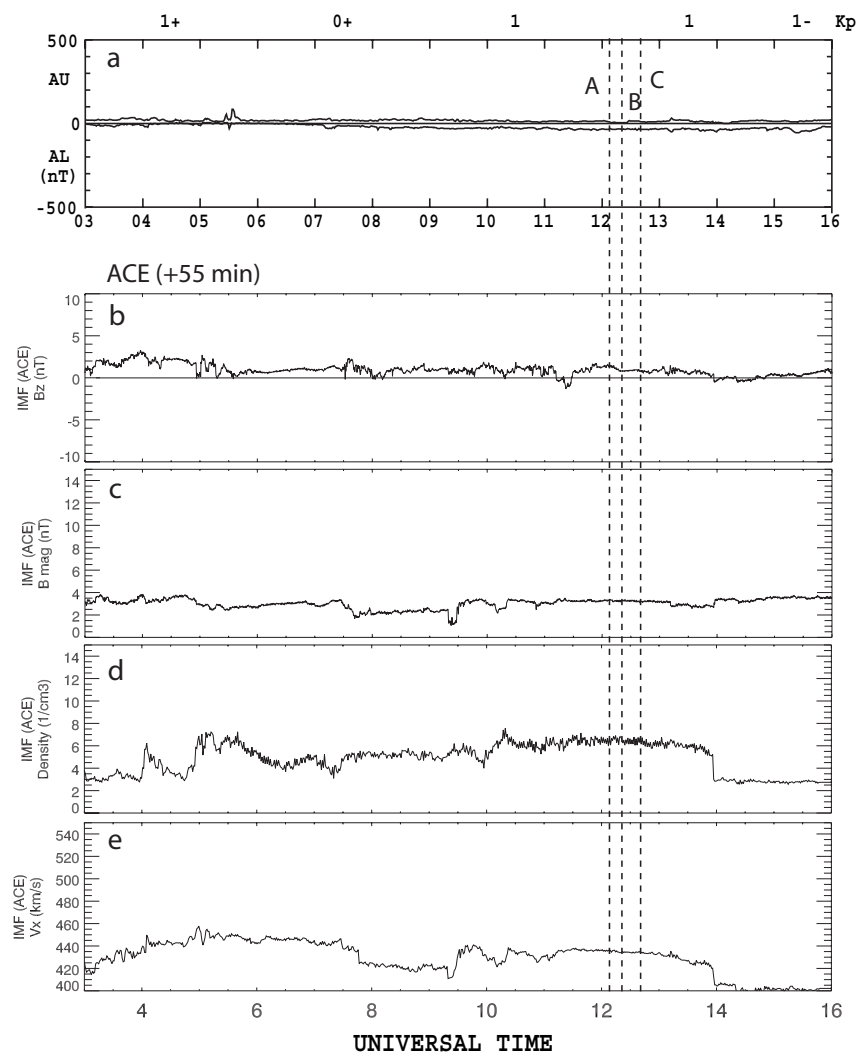

8 September 2002
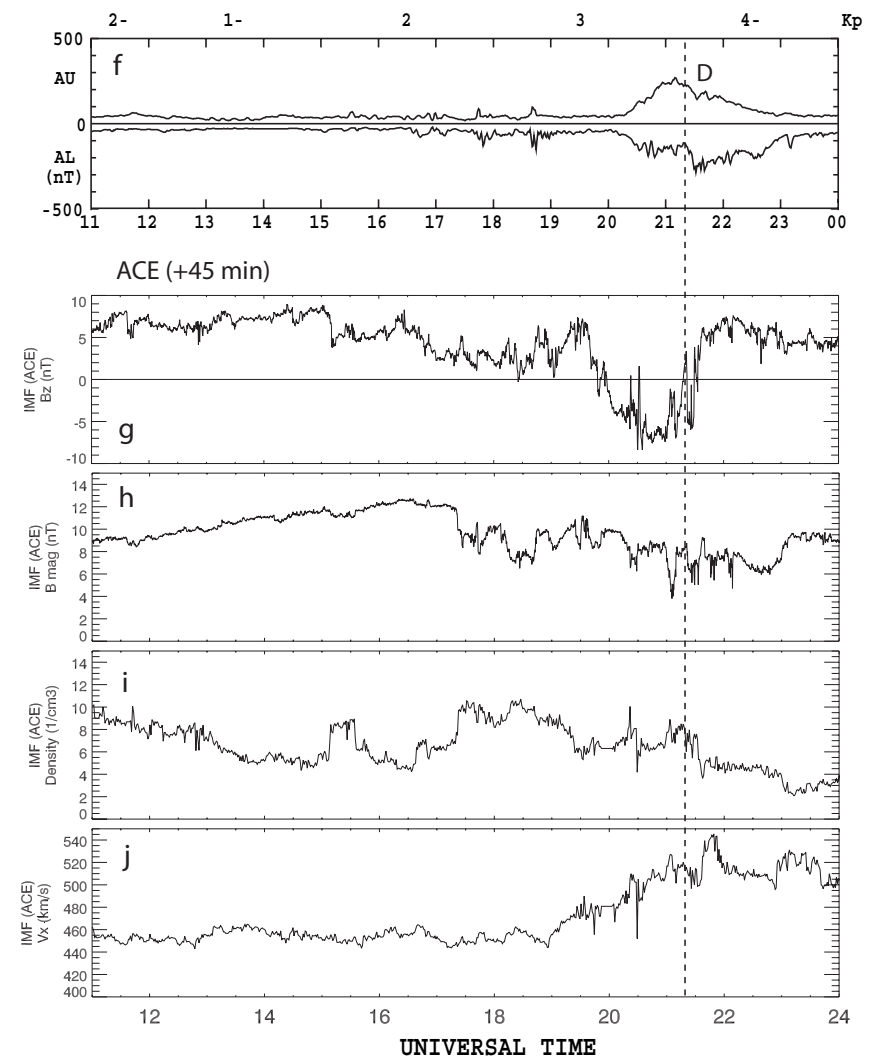

Fig. 5. Solar wind/IMF data and geomagnetic indices to assess the global state of the magnetosphere before and during the events on 24 August 2001 and 8 September 2002. The vertical dashed lines mark the event onsets. (a), (f) $K_{p}$, AU and AL indices from the WDC in Kyoto (Note: These data are quick-look versions). (b-e), (g-j) ACE data showing IMF and solar wind quantities.

of these two satellites either (not shown). It is noted that the local time coverage of the geosynchronous satellites during the events (A, B, and C) on 24 August 2001 was not as optimal as it was for the event (D) on 8 September 2002. In contrast, it was shown in Sect. 2.3 that Geotail located at $\sim 9 R_{E}$ recorded a plasma energization starting with the first event on 24 August 2001. This is in agreement with the thicker current sheet and an expanded dipolar region during this quiet time so that the energization occurred at a distance farther away from geosynchronous orbit and apparently did not reach the geosynchronous satellites.

\subsection{Ground-Cluster-Geotail comparison}

In this section we compare the pulsation activity recorded on the ground with those recorded by Cluster and Geotail. The GIMA ground stations closest to the Cluster footprints on 24 August 2001 are Bettles, Arctic Village and Fort Yukon (Fig. 6, right). Band-pass ( $\mathrm{Pi} 2$ range) filtered magnetic field data $(H$-component) of these three stations are shown in Fig. 8. Data from two additional ground stations (Kaktovik at higher latitude and Ewa Beach at low latitude),
Cluster and Geotail are also shown in this figure. At the time of each Cluster event (A, B and C) Pi2 magnetic pulsations were recorded at all ground stations. A comparison of individual pulses in Fig. 8 shows a good one-to-one correlation between Cluster $B_{x}, B_{z}$ and the three closest stations (see dashed lines) during the events $\mathrm{A}, \mathrm{B}$ and $\mathrm{C}$ except around 12:48 UT. Thus, we conclude from this correlation that the ground Pi2s are related to the Cluster TCRs: either the TCRs caused the ground Pi2s or both had the same source in the tail beyond the location of Cluster $\left(>18 R_{E}\right)$. The only non-correspondence around 12:48 UT is not too surprising considering that there are several deviations among individual ground stations themselves. For example, Arctic Village and Fort Yukon deviate at 12:10 UT (second dashed line from left) in spite of a very good correlation throughout the rest of the interval. Another deviation can, for example, be seen at 12:50 UT between Kaktovik and Arctic Village. It is noted that such differences among ground stations during PBI events have been reported before (e.g. Kim et al., 2005b).

It is important to note that $B_{x}$ was shifted by $50 \mathrm{~s}$ in Fig. 8 , which shows that Cluster was the first to record these 


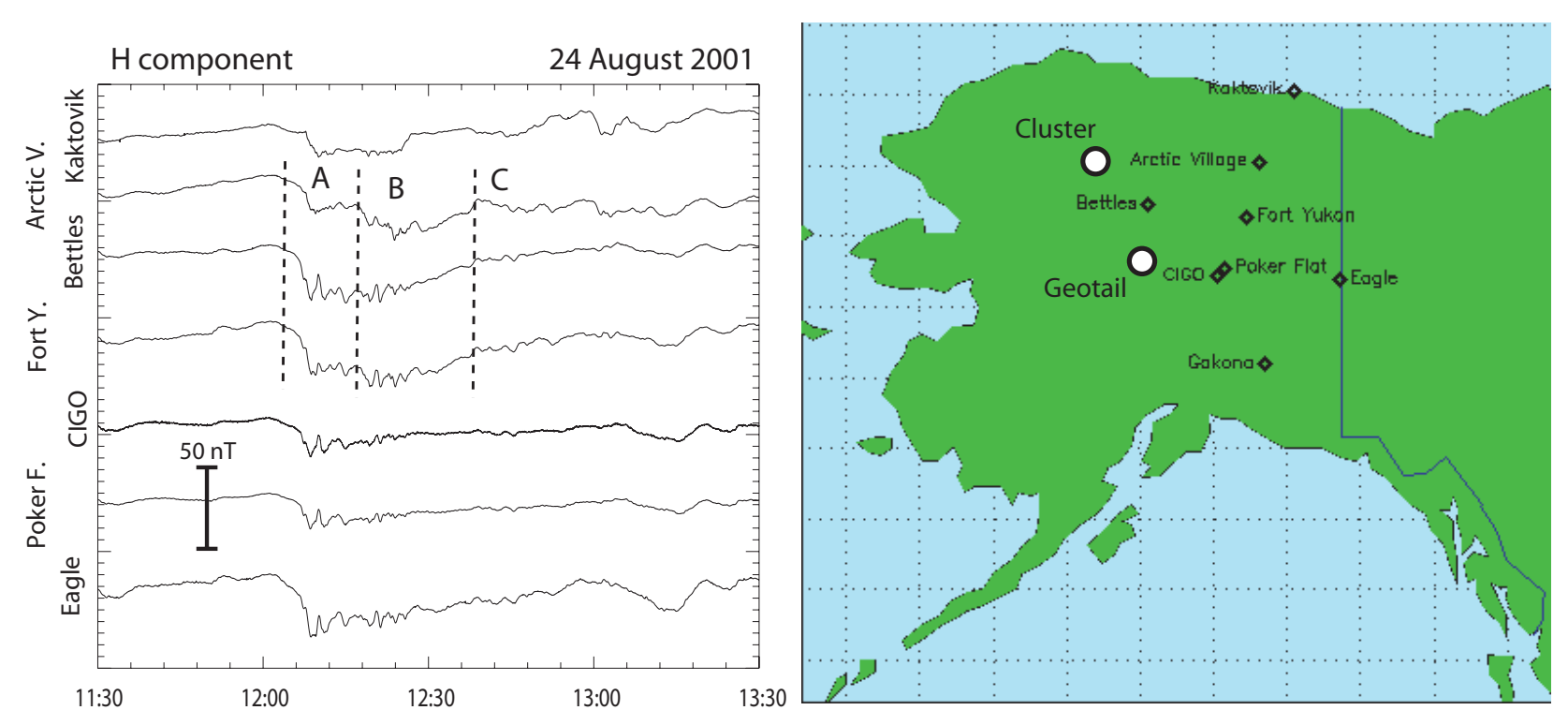

Fig. 6. Right: A geographic map with Cluster's and Geotail's footprints (using T96 for the mapping) together with the GIMA ground stations. Left: Magnetometer data (24 August 2001) from GIMA ground stations. Labels A, B, and C indicate the Cluster events. The dashed lines mark the onsets of small bay-like deflection of the $H$ component.

pulsations. Using Cluster's distance of $\sim 18 R_{E}$ (geocentric), this time delay between Cluster and the ground would correspond to an average propagation speed of $2200 \mathrm{~km} / \mathrm{s}$ for the TCRs to reach the ground. This is consistent with speed estimates of the TCRs from cross-correlation calculations (Sect. 2.1) which yielded a lower limit of $>2000 \mathrm{~km} / \mathrm{s}$. It is also noted that the first four vertical lines (event A) in Fig. 8 deliberately do not line up between Cluster and the ground stations so as to obtain a better correlation. This required shift could be due to a slower propagation speed of event A compared to events B and C.

Whereas the amplitudes of the pulsations increased with each event at Cluster, they remained approximately the same on the ground for events A and B and slightly decreased for event $\mathrm{C}$. This appears to contradict our assertion that the Cluster and ground pulsations have the same source. However, we emphasize again that the smaller amplitudes of the first and second periodic TCR events (A and B) are most likely simply due to the fact that they were recorded farther away from the plasma sheet which in the case of TCR carries the plasma bulge that compresses the lobe field lines. That is, the farther away the spacecraft is from the plasma sheet, the smaller the amplitude of the measured TCR as a general rule. Therefore, it is reasonable to assume that the differences in amplitude between ground and space pulsations is due to this effect.

Figure 8 also includes the low-latitude station Ewa Beach which shows Pi2 starting approximately at the onset time of the $\mathrm{Pi} 2$ recorded at the other stations. The $D$ component is shown because it shows the clearest pulsation signature. It can be noted that the period of the initial pulsations starting at onset (solid line) are longer than those at other stations, and overall the similarity to the Pi2s recorded at other ground stations is less than the similarity between Cluster and the high-latitude ground stations. The main result of interest here is the fact that Pi2s were also observed at low-latitude albeit with somewhat different waveform.

A comparison of the magnetic pulsations recorded by Geotail with those at Cluster (and equivalently those on the ground) is less convincing (Fig. 8). There are several pulses in $B_{x}$ and $B_{z}$ (Geotail) that correlate with the Cluster TCRs but equally many that do not correlate. Overall, the pulsations are, however, of similar periodicity and the onset is comparable to the onset of the first Cluster TCR event (A) albeit delayed by $\sim 50 \mathrm{~s}$. Similarly, the same can be said for the comparison between $B_{x}$ and $B_{z}$ at Geotail and ground pulsations. The best correlation appears to be between $B_{x}$ (Geotail) and Kaktovik. The reader is reminded that Geotail was in the opposite hemisphere at similar local time during this time interval, and, as mentioned in the second paragraph of this sub-section, the ground stations show differences among themselves even though they are located fairly close together. Importantly, one can also identify two intervals of slightly enhanced activity in the Geotail data which coincide with event $\mathrm{A}$ and $\mathrm{B}$; this is not the case for the interval of event $\mathrm{C}$. This might be due to the fact that Geotail was deeper inside the CPS during event $C$ on which we will elaborate in Sect. 3. Nevertheless, we cannot conclude with certainty that the Geotail pulsations are causally related to the ground pulsations, at least not with the same confidence 

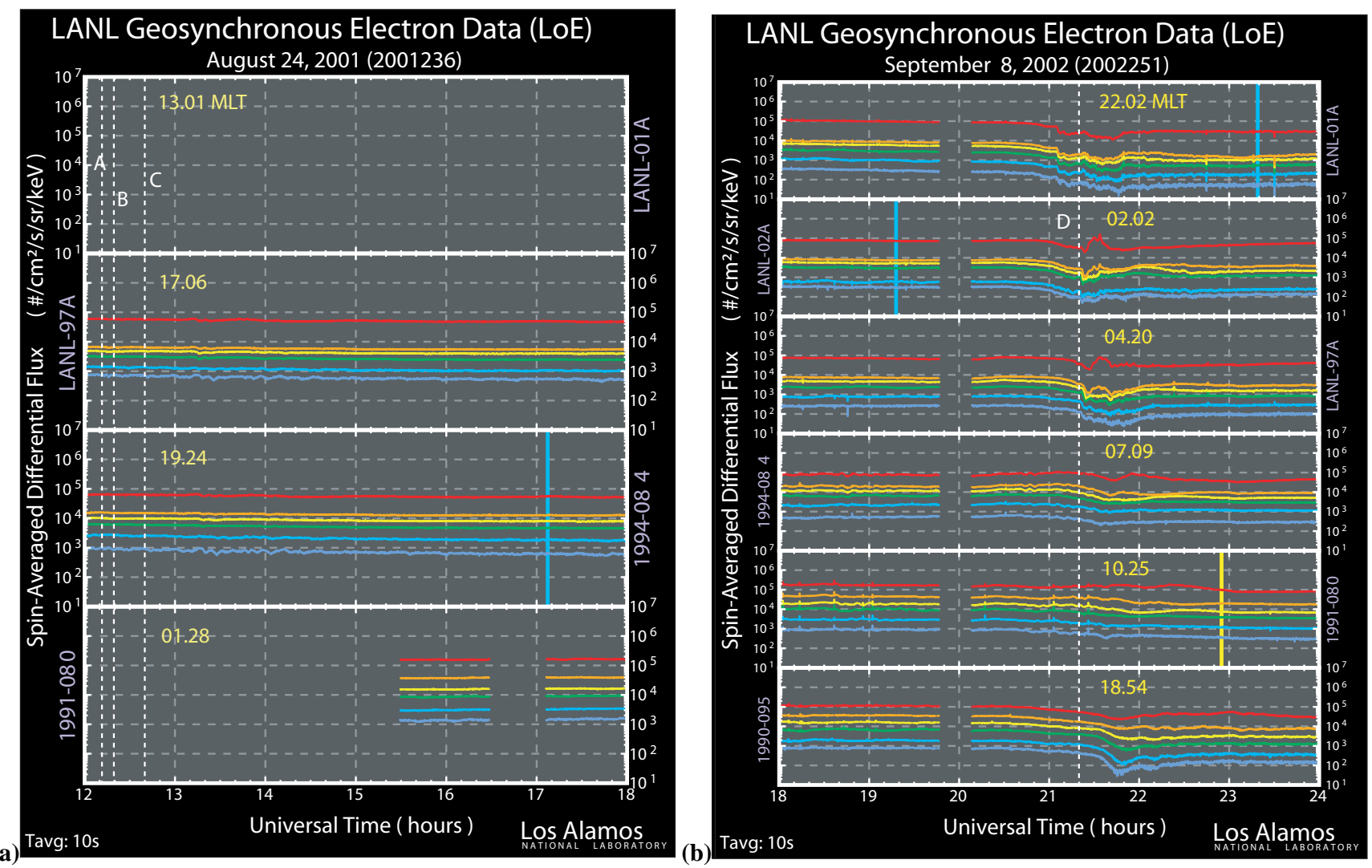

Fig. 7. Energetic electron data from several geosynchronous satellites (LANL) for (a) 24 August 2001 and (b) 8 September 2002 . The vertical dashed lines mark the events A, B, C, and D. The yellow numbers indicate the magnetic local time (MLT) of the satellites at the time of the events $\mathrm{C}$ and $\mathrm{D}$.

as for the Cluster TCRs. Similarly, a comparison (Fig. 8) of velocity moments $\left(V_{x}\right.$ and $\left.V_{y}\right)$ at Geotail with ground pulsations at Cluster is inconclusive and only suggestive that a relationship exists. As for $B_{x}$ and $B_{z}$ (Geotail), one can approximately identify two intervals of enhanced flows with small speeds of less than $80 \mathrm{~km} / \mathrm{s}$ that coincided with the events A and B.

For the 8 September 2002 event, Keiling et al. (2006) reported time-delayed ground $\mathrm{Pi} 2$ at all latitudes (low to high) with the same periodicity and similar waveform as the periodic TCRs recorded by Cluster (see Fig. 10 in Keiling et al., 2006) but less one-to-one correlation with the observations at Geotail. These results were strongly suggestive that the periodic TCRs and the ground Pi2 had the same source. Furthermore, the ground $\mathrm{Pi} 2$ was associated with the onset of a negative substorm $H$ bay, auroral brightening and substorm electrojet intensification, all of which are signatures of substorms.

\section{Discussion}

We reported three events in the tail lobe occurring on $24 \mathrm{Au}-$ gust 2001, two of which were identified as periodic TCRs and the third one was only speculated to be a periodic TCR event. A periodic TCR event is here defined as a sequence of individual TCRs with a repetition rate approximately within the Pi2 frequency range. The three events occurred during very quiet geomagnetic conditions as inferred from the solar wind/IMF and ground data (AU, $\mathrm{AL}, K_{p}$ indices and individual ground magnetometers). Ground magnetometers showed small bay-like disturbances and pulsations in the $H$ component during each periodic TCR event. Such small baylike signatures are associated with PBIs (Lyons et al., 1999; Sutcliffe and Lyons, 2002). Individual pulses of simultaneous ground $\mathrm{Pi} 2$ pulsations were correlated with individual pulses of the periodic TCRs. This allows us to identify the periodic TCRs as lobe Pi2s (similar to the study of Keiling et al., 2006). No particle injection signature was observed at geosynchronous distance (LANL). At $\sim 9 R_{E}$ radial distance (Geotail), the plasma sheet was energized approximately at the onset of the first small non-substorm $H$ bay, 


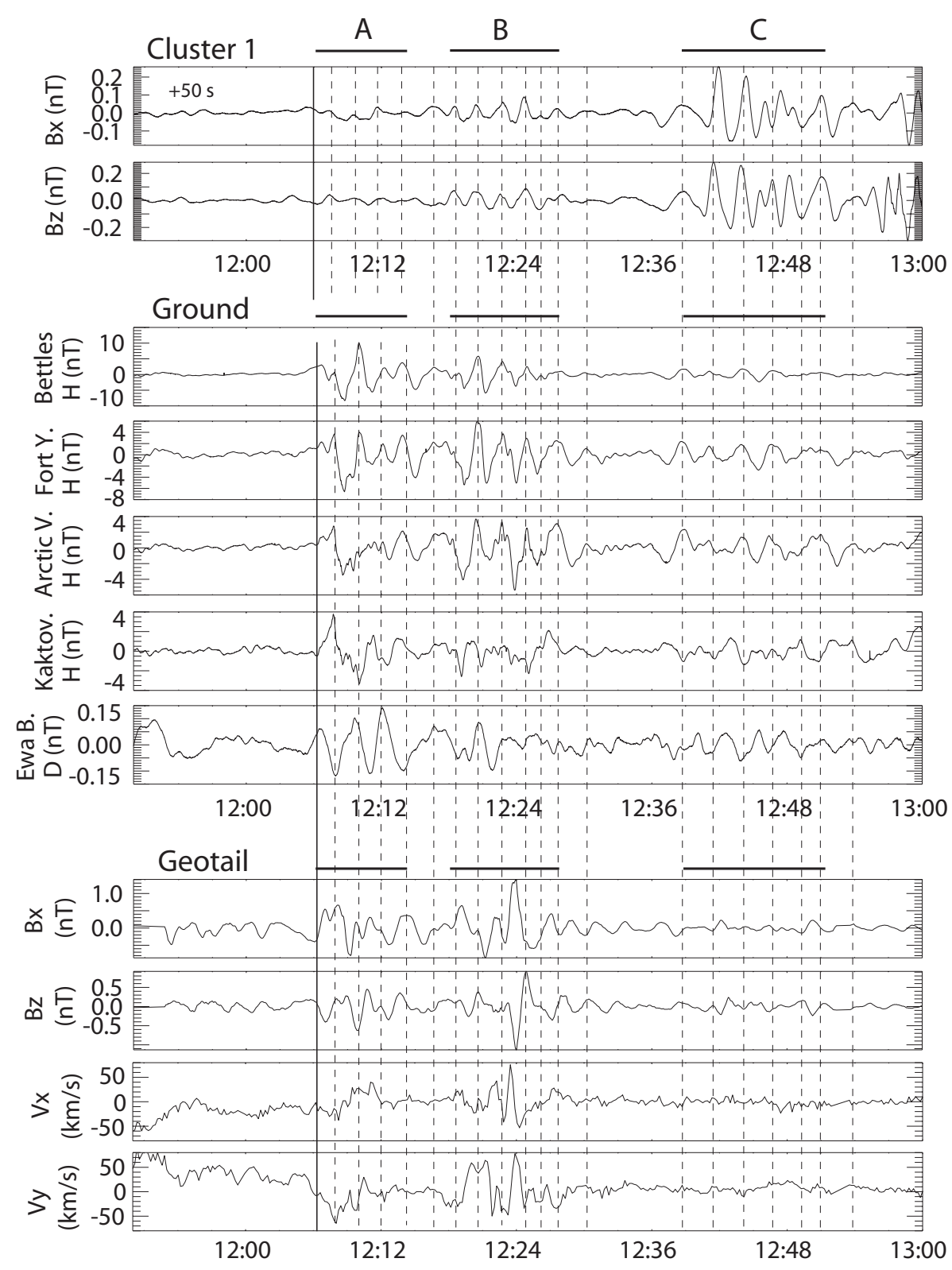

Fig. 8. Comparison of Cluster data, ground data, and Geotail data during the events on 24 August 2001. All data were band-pass filtered $(40 \mathrm{~s}, 150 \mathrm{~s})$ except the flow data of Geotail (last two panels). The ground data are from GIMA stations: Bettles ( $L=6.35)$, Fort Yukon ( $L=6.2$ ), Artic Village $(L=6.47)$, Kaktovik $(L=7.5)$, and the $210 \mathrm{MM}$ station: Ewa Beach $(L=1.6)$. Note that $B_{x}$ of Cluster is shifted by $50 \mathrm{~s}$.

and enhanced magnetic pulsations inside the plasma sheet were observed during the first two events which approximately matched the frequency of the simultaneous ground and Cluster pulsations but no one-to-one correlation existed. Similarly, plasma flow variations at $\sim 9 R_{E}$ were also present during the first two events without one-to-one correlation. Whereas Geotail was closer to the outer edge of the plasma sheet during the first two events, the third event occurred while Geotail was deeper inside the plasma sheet and neither magnetic pulsations nor velocity variations were enhanced during the third event.
We contrasted these quiet-time events (24 August 2001) with a periodic TCR event (8 September 2002) which showed very similar periodicity but occurred under very different global geomagnetic conditions. All investigated signatures indicated a substorm magnetosphere during this event. This substorm-related event was also well correlated with ground Pi2 pulsations (Keiling et al., 2006). In spite of these different global conditions both types of TCRs (quiettime and substorm-related) showed remarkable similarities in their periodic behavior. A direct comparison of the $B_{z}$ component of three events is shown in Fig. 9 (note that all panels 
span the same amount of time, namely $15 \mathrm{~min}$ ). In particular, events $\mathrm{C}$ and $\mathrm{D}$ occurring on two different days showed identical periods (see dashed lines as visual aid). We did not include event A in this figure because it was not definitely confirmed that it was a periodic TCR event.

It has long been established that ground $\mathrm{Pi} 2$ pulsations originate in the magnetosphere where magnetic field oscillations have the same periodicity and waveform as the ground Pi2s (e.g. Takahashi et al., 1995; Osaki et al., 1998). Where in the magnetosphere the characteristic $\mathrm{Pi} 2$ periodicity (40-150 s) appears first and how these oscillations propagate to the ground is however still an area of intense research. Several mechanisms for generating the characteristic Pi2 periodicity have been proposed. Broadly speaking, one can divide them into inner-magnetospheric and outermagnetospheric mechanisms, and evidence for both classes exists. Furthermore, Pi2s occur under a variety of geomagnetic conditions. The Pi2s reported here belong to the class of outer-magnetospheric $\mathrm{Pi} 2$ mechanisms since they were associated with earthward propagating TCRs in the magnetotail, and it was further shown that this type of Pi2 occurs during both quiet geomagnetic conditions in conjunction with PBIs and during active conditions in conjunction with substorms. As was done in Keiling et al. (2006) for the substorm Pi2 event (8 September 2002), we propose that the quiet-time Pi2 events (24 August 2001) were driven with their characteristic period by pulsed reconnection because of their association with TCRs which are believed by some scientists to be the remote signature of reconnection (see Sect. 1). For example, Semenov et al. (2005) showed that the magnetic field perturbations for the 8 September 2002 event are consistent with simulation results of impulsive reconnection events from a single $\mathrm{X}$ line. It is however noted that although the current understanding of the generation mechanism of TCRs is that they are the result of reconnection in the magnetotail, it is not agreed upon whether reconnection is impulsive from possibly a single $\mathrm{X}$ line or occurs simultaneously at multiple locations (e.g. Slavin et al., 2005, and references therein).

Our results also support the suggestion of Kim et al. (2005b) who have studied PBI-Pi2 (i.e. Pi2 occurring in association with PBI). These authored argued on the basis of ground data alone that the Pi2 source which establishes the frequency and duration must have been in the outer magnetosphere for their single event. Our events on 24 August 2001 are similar to theirs, and we show observational evidence in the tail at $18 R_{E}$ that the source must indeed have been at distances greater than $18 R_{E}$. Once again, we emphasize that we do not simply refer to the energy source but rather to the mechanism that controls the Pi2 frequency. Before the study of Kim et al. (2005b), Sutcliffe and Lyons (2002) - reporting on the same event as Kim et al. - already found a correlation between the onset (not the frequency) of PBI-Pi2s and plasma sheet activity in the tail but it was not commented on the source of the characteristic Pi2 frequency. Here we propose that the driver of the PBI-Pi2 frequency on $24 \mathrm{Au}-$
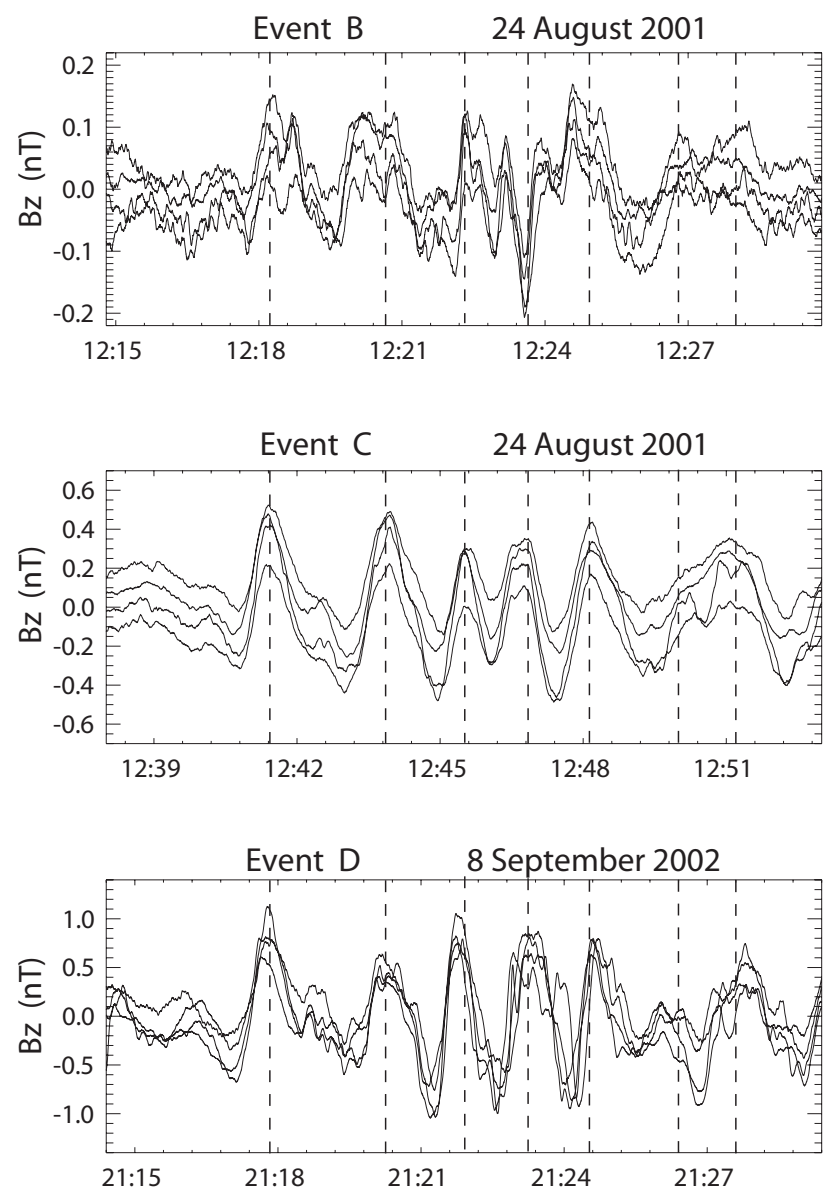

Fig. 9. Cluster $(1,2,3,4)$ magnetic field data $\left(B_{z}\right.$ in GSM) of three periodic TCRs on 24 August 2001 and on 8 September 2002. Data were averaged $(4 \mathrm{~s})$ and detrended (150 s). The vertical dashed lines emphasize the similar periodicity of the events even though they occurred on different days. Note that the time periods of each event cover $15 \mathrm{~min}$ for better comparison.

gust 2001 was pulsed reconnection because of the association with TCRs (see also previous paragraph).

It is surprising that under the very different geomagnetic conditions that prevailed during the two days presented here (24 August 2001 and 8 September 2002) the periodic TCRs had very similar periods two of which were identical (cf. event $\mathrm{C}$ and $\mathrm{D}$ ). What initiated and controlled this periodic reconnection under the very different geomagnetic conditions? The onset of tail reconnection during the substormrelated periodic TCR event on 8 September 2002 was likely triggered by the northward turning of the IMF $B_{z}$ after a period of southward IMF $B_{z}$ (Lyons, 1995). No such trigger was identified for the quiet-time events on 24 August 2001. For the 8 September 2002 event, Semenov et al. (2005) estimated an $\mathrm{X}$ line location at $\sim 30 R_{E}$. In contrast, the thick current sheet prevailing on 24 August 2001 suggests that reconnection occurred much farther than $30 R_{E}$. We also 
reported larger speeds $(>2000 \mathrm{~km} / \mathrm{s}$ ) for the quiet-time TCRs compared to $\sim 700 \mathrm{~km} / \mathrm{s}$ for the substorm-related TCRs. A larger data base is necessary to determine whether this large speed is characteristic for quiet-time periodic TCRs. These additional differences do not make it easier to answer the question posed in this paragraph. At the moment no explanation can be given for the mechanism causing the "pulsation" of reconnection (provided that pulsed reconnection was indeed operating) during both very different geomagnetic conditions. It is noted, however, that the time scale present in the here reported events can in fact be found not only for Pi2 pulsations but for other magnetospheric phenomena as well (see Sergeev et al., 1996); for example, the formation of successive poleward arcs occurs every 1-3 min, proton injections at geosynchronous orbit have an average repetition time of $2.6 \mathrm{~min}$, and the repetition time of bursts in a bursty bulk flow event is $1-3 \mathrm{~min}$. These phenomena have been linked to impulsive reconnection albeit during substorms. Here we showed this repetition rate during a geomagnetically very quiet time.

Another important question is: How did the Pi2 disturbance travel from the possible reconnection site to the ground where ground Pi2 pulsations were observed? Keiling et al. (2006) proposed two scenarios for a physical link between the tail and ground observations. One scenario required for the TCR to travel from the reconnection site through the PSBL/lobe to the ground causing directly the Pi2 pulsations; the other scenario was modeled after the BBF-Pi2 model of Kepko et al. (2001) where an associated periodic BBF disturbance traveled through the central plasma sheet. In this latter scenario the BBFs propagate to the near-Earth region where they lead to dipolarization and subsequently the expansion of the thin plasma sheet. The braking of a BBF would generate an inertial current (e.g. Shiokawa et al., 1998). Kepko et al. (2001) thus suggested that braking of periodic BBFs can lead to periodic inertia currents which are diverted to the high-latitude ionosphere where they cause ground Pi2. Although they suggested that the ground pulsations would be of small amplitudes (few nT), Keiling et al. (2006) showed for the 8 September 2002 event that the amplitudes could reach several tens of nT. Furthermore, TCRs have been associated with BBFs in the central plasma sheet (e.g. Slavin et al., 2003a). The speed of $\sim 700 \mathrm{~km} / \mathrm{s}$ for the substorm TCRs is a reasonable speed of BBFs; however, the much larger speeds for the quiet-time TCRs of $>2000 \mathrm{~km} / \mathrm{s}$ is less likely for BBFs but still possible. Although the Geotail observations at $9 R_{E}$ on 24 August 2001 showed very small flow velocities, they were much smaller than those reported by Kepko et al. (2001). However, we cannot rule out that the flow braked farther away from Geotail which is plausible considering the thick current sheet on this day (i.e. extended dipolar region). Therefore, Geotail might simply not have been located suitably to record the flow bursts. Furthermore, it is also possible that the first scenario (i.e. propagation through the PSBL) is applicable since the magnetic pulsations and flow velocity variations at Geotail were in fact more pronounced closer to the outer edge of the plasma sheet for the first two events (events A and B) and not observed deeper inside the central plasma sheet during the third event (event C). This rather suggests that the disturbances propagated near the outer edge which is consistent with the fact that the correlated Pi2 occurred at high-latitudes $(L>6)$ in concert with the high-latitude phenomenon of PBIs. In spite of these observations and lack thereof, we cannot conclusively answer the question posed in this paragraph.

An important observation is the simultaneous occurrence of low-latitude ( $L=1.6$ ) Pi2. Although the period and waveform were different compared to the high-latitude $(L>6) \mathrm{Pi} 2$, it shows that the Pi2 disturbance was observed over many $L$ values in association with the lobe Pi2 (periodic TCRs). Both Sutcliffe and Lyons (2002) and Kim et al. (2005b) reported similar low-latitude Pi2 in concert with a high-latitude PBI-Pi2. Furthermore, Kim et al. (2005b) reported frequency variations of the Pi2 observed at different latitudes and longitudes during the event. The lack of a pronounced Pi2 signature deeper in the central plasma sheet (at $9 R_{E}$ ) during our event seems to rule out or at least does not suggest that the low-latitude Pi2 was caused by a disturbance which propagated near the equatorial plane all the way from the distant source to the ground.

Finally, we propose the possibility that the compressional fluctuations of event A which do not show all characteristics of TCR might in fact also be periodic TCRs. Most studies of TCRs only consider fairly large compression ratio of $>1 \%$, thus excluding many TCRs that are located farther away from the plasma sheet and therefore have smaller compression ratios. In such cases it is more likely that the 3-D shape of the compression region plays a controlling factor on what components are observed strongest, and it is imaginable (no numerical simulation has been done here) that under those conditions the other TCR criteria are not as clear. Furthermore, the occurrence of event $\mathrm{A}$ at the time of a small $\mathrm{H}$ bay-like disturbance similar to the other two events (B and C) supports the view that indeed event A was generated by the same mechanism.

\section{Conclusions}

The main conclusions of this study are:

1. We have given further evidence that periodic TCRs observed in the tail lobe at distances of $18 R_{E}$ and ground Pi2 are related phenomena (for some type of Pi2). In particular, it was shown that both had the same periodicity and occurred simultaneously (allowing for propagation time delays) strongly suggesting that they had the same source. Since the TCRs were propagating Earthward, the source of both periodic TCRs and ground Pi2 was located in the outer magnetosphere beyond $18 R_{E}$. 
2. We have shown that the association of periodic TCRs and ground Pi2 also holds during very quiet geomagnetic conditions in addition to the previous result which showed this association during substorms (Keiling et al., 2006). Furthermore, this quiet time was identified as a period of PBIs on the basis of small ( $<40 \mathrm{nT}) H$-baylike signatures at high-latitude ground stations.

3. Combining (1) and (2), our results give direct observational evidence that the frequency of PBI-associated ground $\mathrm{Pi} 2$ is controlled in the distant tail. We propose that the controlling mechanism is pulsed reconnection, which has not been proven here but is based on previous results (Semenov et al., 2005; Keiling et al., 2006) which combined model results and observations of substorm-related periodic TCRs and associated ground Pi2. It is important to note that in this scenario reconnection not only provided the energy but its temporal variation also controlled the characteristic $\mathrm{Pi} 2$ frequency.

4. Quiet-time and substorm periodic TCRs can have identical periods. If they are indeed generated by pulsed reconnection (or possibly multiple $\mathrm{X}$ lines), it raises the question of what causes this identical periodic behavior under very different geomagnetic conditions. This question could not be answered in this study but it demonstrates the need for further investigations of periodic TCRs (lobe Pi2s) with a larger data set that should further clarify the connection of lobe Pi2s to both the reconnection region and ground $\mathrm{Pi} 2 \mathrm{~s}$.

5. We have shown that TCRs with small compression ratios $(\Delta B / B<1 \%)$ can be useful to study, and we propose that other compressional fluctuations with $\Delta B / B<1 \%$ seen in the tail lobe could possibly be related to the same mechanism that generates TCRs with $\Delta B / B>1 \%$ (which are more commonly studied). The smaller compression ratios are in some cases due to larger observation distances from the plasma sheet, thus resulting in signatures that are possibly more difficult to identify as TCRs.

Acknowledgements. This work was supported by NASA grant NNG05GL27G-06/08. The geomagnetic indices were provided by the World Data Center for Geomagnetism, Kyoto (http://swdcwww. kugi.kyoto-u.ac.jp). We thank D.-L. Wilkinson and K. Yumoto for providing magnetometer data from the Geophysical Institute Magnetometer Array (GIMA) and the $210^{\circ}$ Magnetic Meridian network $(210 \mathrm{MM})$, respectively. The Geotail data were obtained through the Coordinated Data Analysis Web (CDAWeb) and DARTS at the Institute of Space and Astronautical Science (ISAS), Japan. We acknowledge the data providers D. Williams (APL/JHU) for the EPIC data, S. Kokubun (STELAB) for the MGF data, and T. Mukai (ISAS) for the LEP data. Thanks is also due to the reviewers for their critical comments.

Topical Editor I. A. Daglis thanks C. Jacquey and another anonymous referee for their help in evaluating this paper.

\section{References}

Baker, D. N., Hones Jr., E. W., Higbie, P.R., et al.: Global properties of the magnetosphere during a substorm growth phase: A case study, J. Geophys. Res., 86, 8941-8956, 1981.

Henderson, M. G., Reeves, G. D., and Murphree, J. S.: Are northsouth aligned auroral structures an ionospheric manifestation of bursty bulk flows?, Geophys. Res. Lett., 25, 3737-3740, 1998.

Keiling, A., Fujimoto, M., Hasegawa, H., et al.: Association of Pi2 pulsations and pulsed reconnection: ground and Cluster observations in the tail lobe at $16 R_{E}$, Ann. Geophys., 24, 3433-3449, 2006,

http://www.ann-geophys.net/24/3433/2006/.

Kepko, L. and Kivelson, M. G.: Generation of $\mathrm{Pi} 2$ pulsations and bursty bulk flows, J. Geophys. Res., 104, 25 021-25 034, 1999.

Kepko, L., Kivelson, M. G., and Yumoto, K.: Flow bursts, braking, and Pi2 pulsations, J. Geophys. Res., 106, 1903-1916, 2001.

Kim, K.-H., Lee, D.-H., Takahashi, K., Russell, C. T., Moon, Y.-J., and Yumoto, K.: Pi2 pulsations observed from the Polar satellite outside the plasmapause, Geophys. Res. Lett., 32, L18102, doi:10.1029/2005GL023872, 2005a.

Kim, K.-H., Takahashi, K., Lee, D.-H., Sutcliffe, P. R., and Yumoto, K.: Pi2 pulsations associated with poleward boundary intensifications during the absence of substorms, J. Geophys. Res., 110, A01217, doi:10.1029/2004JA010780, 2005b.

Lee, D.-H. and Lysak, R. L.: MHD waves in a three-dimensional dipolar magnetic field: A search for Pi2 pulsations, J. Geophys. Res., 104, 28 691-28 699, 1999.

Lyons, L. R.: A new theory for magnetospheric substorms, J. Geophys. Res., 100, 19069-19081, 1995.

Lyons, L. R., Nagai, T., Blanchard, G. T., et al.: Associations between Geotail plasma flows and auroral poleward boundary intensifications observed by CANOPUS photometers, J. Geophys. Res., 104, 4485-4500, 1999.

Moldwin, M. B. and Hughes, W. J.: Observations of earthward and tailward propagating flux rope plasmoids: Expanding the plasmoid model of geomagnetic substorms, J. Geophys. Res., 99, 184-198, 1994.

Osaki, H., Takahashi, K., Fukunishi, H., Nagatsuma, T., Oya, H., Matsuoka, A., and Milling, D. K.: Pi2 pulsations observed from the Akebono satellite in the plasmasphere, J. Geophys. Res., 103, 17 605-17 615, 1998.

Reeves, G. D., Fritz, T. A., Cayton, T. E., and Belian, R. D.: Multisatellite measurements of the substorm injection region, Geophys. Res. Lett., 17, 2015-2018, 1990.

Semenov, V. S., Kubyshkin, I. V., Lebedeva, V. V., et al.: A comparison and review of steady-state and time-varying reconnection, Planet. Space Sci., 40, 63-87, 1992.

Semenov, V. S., Penz, T., Ivanova, V. V., et al.: Reconstruction of the reconnection rate from Cluster measurements: First results, J. Geophys. Res., 110, A11, doi:10.1029/2005JA011181, 2005.

Sergeev, V. A., Bosinger, T., Belgian, R. D., Reeves, G. D., and Cayton, T. E.: Drifting holes in the energetic electron flux at geosynchronous orbit following substorm onset, J. Geophys. Res., 97, 6541-6548, 1992a.

Sergeev, V. A., Elphic, R. C., Mozer, F. S., et al.: A two-satellite study of nightside flux transfer events in the plasma sheet, Planet. Space Sci., 40, 1551-1572, 1992b.

Sergeev, V. A., Pulkkinen, T. I., and Pellinen, R. J.: Coupled-mode scenario for the magnetospheric dynamics, J. Geophys. Res. 
101, 13 047-13 065, 1996.

Sergeev, V. A., Kubyshkina, M. V., Baumjohann, W., et al.: Transition from substorm growth to substorm expansion phase as observed with a radial configuration of ISTP and Cluster spacecraft, Ann. Geophys., 23, 2183-2198, 2005, http://www.ann-geophys.net/23/2183/2005/.

Shiokawa, K., Baumjohann, W., Haerendel, G., et al.: High-speed ion flow, substorm current wedge, and multiple Pi2 pulsations, J. Geophys. Res., 103, 4491-4507, 1998.

Slavin, J. A., Smith I, E. J., Tsurutani, B. T., et al.: Substorm associated traveling compression regions in the distant tail: ISEE-3 geotail observations, Geophys. Res. Lett., 11, 657-660, 1984.

Slavin, J. A., Smith, M. F., Mazur, E. L., et al.: ISEE 3 Observations of traveling compression regions in the Earth's magnetotail, J. Geophys. Res., 98, 15 425-15 446, 1993.

Slavin, J. A., Lepping, R. P., Gjerloev, J., et al.: Geotail observations of magnetic flux ropes in the plasma sheet, J. Geophys. Res., 108(A1), 1015, doi:10.1029/2002JA009557, 2003a.

Slavin, J. A., Owen, C. J., Dunlop, M. W., et al.: Cluster four spacecraft measurements of small traveling compression regions in the near-tail, Geophys. Res. Lett., 30(23), 2208, doi:10.1029/2003GL018438, 2003b.

Slavin, J. A., Tanskanen, E. I., Hesse, M., et al.: Cluster observations of taveling compression regions in the near-tail, J. Geophys. Res., 110, A06207, doi:10.1029/2004JA010878, 2005.
Sutcliffe, P. R.: Observations of Pi2 pulsations in a near ground state magnetosphere, Geophys. Res. Lett., 25, 4067-4070, 1998.

Sutcliffe, P. R. and Lyons, L. R.: Association between quiettime Pi2 pulsations, poleward boundary intensification, and plasma sheet particle fluxes, Geophys. Res. Lett., 29, 4067-4070, doi:10.1029/2001GL014430, 2002.

Taguchi, S., Slavin, J. A., and Lepping, R. P., et al.: IMP 8 observations of traveling compression regions in the mid-tail near substorm expansion phase onset, Geophys. Res. Lett., 24, 353356, 1997.

Taguchi, S., Slavin, J. A., Kiyohara, M., et al.: Temporal relationship between midtail traveling compression regions and substorm onset: Evidence for near-Earth neutral line formation in the late growth phase, J. Geophys. Res., 103(A11), $26607-$ $26612,1998$.

Takada, T., Nakamura, R., Baumjohann, W., et al.: Do BBFs contribute to inner magnetosphere dipolarizations: Concurrent Cluster and Double Star observations, Geophys. Res. Lett., 33, L21109, doi:10.1029/2006GL027440, 2006.

Takahashi, K., Ohtani, S., and Anderson, B. J.: Statistical analysis of Pi2 pulsations observed by AMPTE CCE spacecraft in the inner magnetosphere, J. Geophys. Res., 100, 21 929-21 942, 1995.

Yumoto, K., and the 210 degree MM magnetic observation group: The STEP 210 degree magnetic meridian network project, J. Geomag. Geoelectr., 48, 1297-1309, 1996. 